\title{
Ancient Eclipses and the Earth's Rotation
}

\section{L.V. Morrison}

28 Pevensey Park Road, Westham, Pevensey, East Sussex, UK

\author{
F.R. Stephenson \\ Physics Dept., University of Durham, South Road, Durham, UK
}

\begin{abstract}
The analysis of historical eclipses shows that the length of the day is increasing on the average by 1.8 milliseconds per century, as distinct from the value of 2.3 milliseconds per century expected from tidal friction. The difference may be accounted for by the response of the Earth to post-glacial uplift following the end of the last ice-age.
\end{abstract}

\section{Tidal acceleration of the Moon}

Until fairly recently there were two main unknowns in the analysis of ancient eclipses: the tidal acceleration of the Moon and long-term changes in the rotation of the Earth. These were highly correlated and all attempts to separate them were unsatisfactory, which resulted in unreliable estimates for both unknowns, even though some of the data were potentially of good intrinsic accuracy. Fortunately, as a result of laser-ranging to the Moon and artificial satellites, we now have good estimates of the tidal acceleration of the Moon and the corresponding tidal braking of the Earth's rotation. The tidal acceleration of the Moon is close to $-26 \operatorname{arcsec} /\left(\right.$ century) ${ }^{2}\left({ }^{\prime \prime} / \mathrm{cy}^{2}\right)$ (Williams et al. 1992) and we adopt this value and solve for changes in the rotation of the Earth. The full potential of the eclipse observations can then be realised.

\section{Records of eclipses}

From the point of view of measuring changes in the rotation of the Earth, eclipses can be divided into two main categories:

1. untimed $=$ eyewitness accounts of total solar and lunar eclipses;

2. timed $=$ measurement of time of day or night of some aspect of an eclipse.

For untimed eclipses the essential requirements are that the account contains an unambiguous description of totality, and the date and place of observation. The calculation of the track of totality is made on the assumption of a constant rate of rotation of the Earth and a tidal acceleration of $-26 " / \mathrm{cy}^{2}$ for the Moon. The difference in longitude between the calculated and observed tracks, converted to time, is a measure of the difference at that epoch between the time on the 


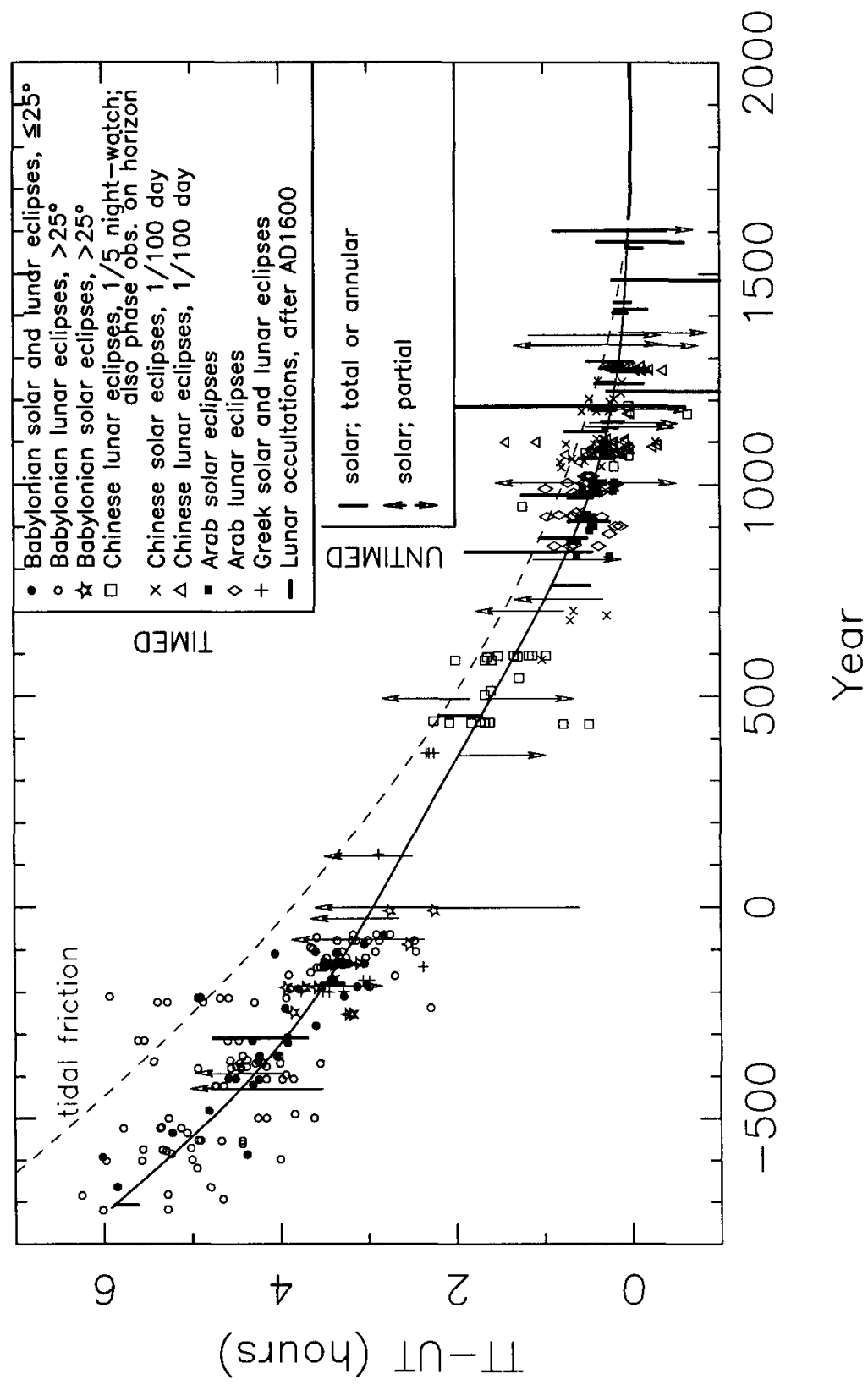

Figure 1. Plot of the difference, TT-UT, from the collected untimed and timed observations of eclipses -719 to +1605 . The continuous curve is fitted by cubic splines to the data. Note that the vertical lines are not error bars, but solution space, anywhere in which the actual value is equally likely to lie. Arrowheads denote that the solution space extends several hours in that direction. 
uniform time-scale Terrestrial Time (TT) and the Earth's rotational time-scale Universal Time (UT). The uncertainty of the observation is the width of the track at the observer, projected parallel to the equator. This can be as little as a few minutes or greater than an hour, where the track is nearly parallel to the equator. The actual position of the observer, and hence the Earth, can be anywhere within the track width, giving a range of values for the difference TT-UT; but the limits of the range are well-defined, as a result of the sharp boundaries of totality.

In timed observations the Babylonians measured (probably with a clepsydra) the time elapsed between sunset or sunrise and some aspect of a lunar eclipse, usually first or last contact. The elapsed time, added/subtracted to the known time of sunset/sunrise gives the UT of the observation. The dominant uncertainty here is in measuring the elapsed time with a water clock. However, there are circumstances when the eclipse begins very soon after sunset, and the clock error is thereby reduced. In the limiting case where the eclipse begins at or before sunset, the observation can be treated as in the first category above, relying on the geometry of the situation, and not the timing. The range of solution is, of course, generally much greater than in the timing of the eclipse which produces a discrete value of TT-UT. In the case of Chinese observations, the time of an eclipse is given as a fraction of the day or night. In Arab observations, the time is determined by the altitude of the Sun or a reference star.

Records of eclipses from the civilizations of ancient Babylon, China, the Arab Dominions and ancient and medieval Europe which are useful in the study of the Earth's rotation have been collected, translated and published by Stephenson (1997), and analysed by Stephenson \& Morrison (1995). Here we report on the latest results obtained by adding more observations to those in our 1995 paper.

\section{Analysis of TT-UT and the deduced changes in the lod}

The timed and untimed datasets for TT-UT are independent and are analysed separately. However, for brevity the two datasets are plotted in one figure here (Figure 1). The untimed lunar data, which are less important than the solar data, are omitted to make the figure less crowded. The expected overall behaviour of TT-UT is parabolic with time, as a result of the tidal torque operating on the Earth, and this is borne out in Figure 1. However, a simple parabola does not satisfy all the constraints of the data, as discussed in Stephenson \& Morrison (1995), and a cubic spline is fitted with the sparing use of knots to smooth the data, compatible with the inaccuracies exhibited by their vertical dispersion at each epoch. The fluctuations in the spline fit are seen more clearly in the first time-derivative which gives the change in the length of the day (lod) plotted in Figure 2.

The change in the lod, which expresses the change in the rate of rotation of the Earth, is measured here in units of milliseconds (ms), and is the change with respect to the standard day of $86400 \mathrm{~s}$ SI. The rate of change in the lod, which expresses the acceleration, is measured in ms per century (ms/cy).

The expected change of $+2.3 \mathrm{~ms} / \mathrm{cy}$ due to tidal braking is calculated from the results of Christodoulidis et al. (1988) with a value of $-26^{\prime \prime} / \mathrm{cy}^{2}$ for the 


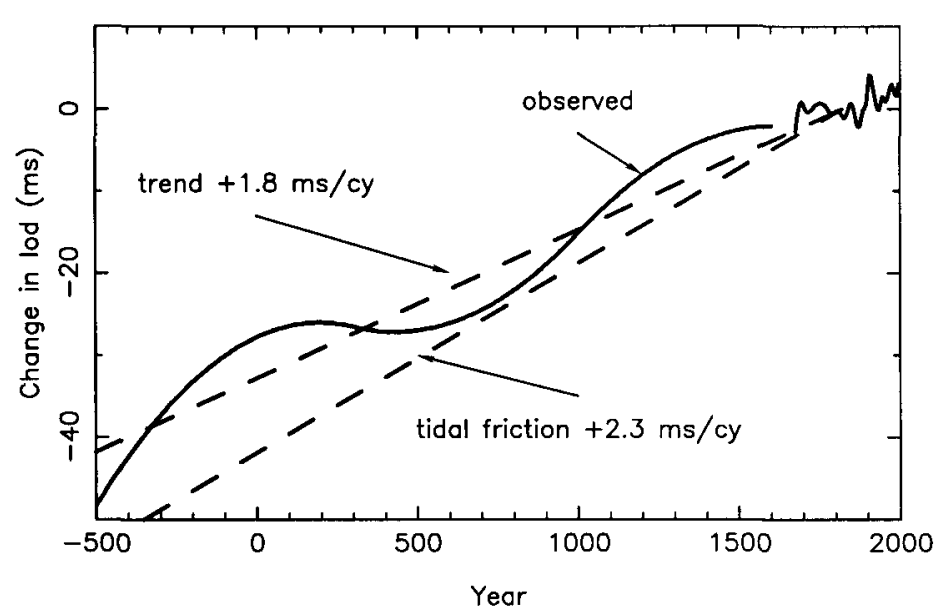

Figure 2. Plot of the changes in the length of day (lod) -500 to +1996 obtained by taking the first time-derivative along the spline curve shown in Figures 1 . The change due to tidal friction is $+2.3 \mathrm{~ms} / \mathrm{cy}$. The high-frequency changes in the lod after AD 1700 are taken from Stephenson \& Morrison (1984).

tidal acceleration of the Moon. It is clear from Figure 2 that there is another significant component in the Earth's rotation over the past 2500 years besides that due to tidal friction. This non-tidal component, which produces an acceleration of the Earth's rotation, is most likely to arise from a secular change of the Earth's zonal harmonic $J_{2}$ due to post-glacial rebound of the lithosphere since the last ice-age (Peltier et al. 1983). Analysis by Cheng et al. (1989) of Starlette data leads to a rate of $-0.44 \pm 0.05 \mathrm{~ms} / \mathrm{cy}$ in the lod. Taken from the tidal rate, this predicts a secular trend of $+1.8 \mathrm{~ms} / \mathrm{cy}$. This agrees with the 'trend' drawn through the observed fluctuations in the lod shown in Figure 2.

The long-term fluctuations have an amplitude of about $4 \mathrm{~ms}$, which is comparable to those of the decade fluctuations after AD 1700 deduced by Stephenson \& Morrison (1984) from lunar occultations. The decade fluctuations are probably present throughout the whole period displayed in Figure 2, but their frequency is too high to be resolved by the eclipse data. The long-term fluctuations in Figure 2 may be the low frequency end of changes caused by coremantle coupling, as discussed by Lambeck (1980). More observations in the period AD 100-700, as yet undiscovered, would help to define these fluctuations.

\section{References}

The references cited in this necessarily brief paper may be found in the following: Stephenson, F.R. 1997, Historical eclipses and Earth's rotation (Cambridge: Cambridge University Press)

Stephenson, F.R., \& Morrison, L.V. 1995, Long-term fluctuations in the Earth's rotation: 700 BC-AD 1990, Phil. Trans. R. Soc. Lond. Ser. A, 351, 165 